\title{
Metastatic prostate cancer cell-specific phage-like particles as a targeted gene-delivery system
}

\author{
Olusegun A Fagbohun ${ }^{1,2,4}$, Robert A Kazmierczak ${ }^{1,2}$, Valery A Petrenko ${ }^{3}$ and Abraham Eisenstark ${ }^{1,2^{*}}$
}

\begin{abstract}
Background: One of the cardinal requirements for effective therapeutic management of tumors is the selective delivery of cancer drugs to the right site by ligand-decorated nanomedicines. Screening of $2 \times 10^{9}$ clone landscape phage library provides a reliable avenue for generating protein ligands specific for tumor cells. It was shown that selective phage proteins derived from landscape phage libraries against breast and prostate cancer cells are able to navigate drug or siRNA loaded liposomes to corresponding cancer cells with minimal toxicity to non-neoplastic cells. In an alternative platform, glioma cell-specific phage proteins were used for assembling in vivo cancer-specific phage-like particles, named 'phagemid infective particles' as targeted gene-delivery vehicles.

Methods: To extend the panel of anticancer cell phages, we have screened a $2 \times 10^{9}$ clone landscape phage library f8/8 to select phage clones specific for metastatic prostate cancer cell PC-3M. The phage clones were characterized for their selective interaction with PC-3M cells using phage capture assay, immunofluorescence microscopy and electron microscopy. A prostate cancer selective phage was converted to phage-like particles harboring emerald green fluorescent protein.
\end{abstract}

Results: Phage clone EPTHSWAT (designated by the sequence of inserted peptide) was found to be most selective for PC-3M cells and was observed to internalize PC-3M cells as revealed by immunofluorescence microscopy and electron microscopy. Conversion of this phage to phage-like particles harboring emerald green fluorescent protein and the expression of emerald green fluorescent protein in the phage-like particles treated PC-3M cells showed potential of adoption of this phage-like particle in prostate cancer therapeutic gene delivery.

Conclusion: Successful employment of phage-like particles expressing emerald green fluorescent protein genes targeted to prostate cancer cells PC-3M confirms a prospect of their use for targeted delivery of therapeutic genes to cancer cells.

Keywords: Phage-like particles, Prostate cancer, Phage, Gene-delivery

\section{Background}

Prostate cancer is the second most frequently diagnosed cancer amongst men in the US. An estimated 238,590 new cases are estimated to be diagnosed with an expected death of 29,720 in men in the U.S in 2013 [1]. Conventional treatment modalities based on surgery, radiotherapy and chemotherapy have not ensured complete remission of the disease, which would account for $28 \%$ incidence of cancers of men in the U.S in 2013 [1]. Therefore, new concepts of prostate cancer care, including therapeutic oligoand polynucleotides have been proposed as a promising

\footnotetext{
* Correspondence: EisenstarkA@missouri.edu

${ }^{1}$ Cancer Research Center, Columbia, MO 65201, USA

2University of Missouri, Columbia, MO 65211, USA

Full list of author information is available at the end of the article
}

alternative [2]. However, a prerequisite for effective gene therapy is specific delivery of therapeutic genes to a target tissue and its sustained expression in the effected cancer cells [3]. Current gene delivery approaches are based on the use of viral and non-viral delivery systems. Non-viral systems comprise naked DNA, or DNA associated with polymers, dendrimers, liposomes, polyethylenimine, polylysine, cationic peptides and, other non-infective vehicles. While non-viral delivery systems assure safety and modularity $[4,5]$, they rank in efficiency of cell transduction below mammalian viral therapeutic oligonucleotides delivery vesicles based on herpesviruses, retroviruses, adenoviruses, or adeno-associated viruses, which have been evolved to acquire natural tropism for mammalian cells.

\section{Biomed Central}

(c) 2013 Fagbohun et al.; licensee BioMed Central Ltd. This is an Open Access article distributed under the terms of the Creative Commons Attribution License (http://creativecommons.org/licenses/by/2.0), which permits unrestricted use, distribution, and reproduction in any medium, provided the original work is properly cited. 
The natural high transduction activity of viral vehicles, however can limit their use as therapeutics, since they can readily infect both target tumor cells and non-related healthy cells and can provoke fulminant immune response. Thus, they require reengineering to extirpate their natural tropism, re-target to specific tissues and make them less immunogenic [6,7]. By contrast, filamentous bacteriophages (phages) which are prokaryotic viruses, possess no natural tropism for mammalian cells, yet can be adapted to evolve tropism for target tumor cells by fusion of their surface proteins with tumor-specific peptides $[8,9]$. For example, phages with fusion peptides as targeting ligands on the pIII proteins have been shown to transduce mammalian cells with subsequent transgene delivery [10-13]. In another study, [14] developed phagemid particles with epidermal growth factors fused to pIII to transduce mammalian cells. Another type of display, with fusion peptides displayed on all multiple copies of the major coat protein pVIII can be even more advantageous for cell transduction since the multivalent display results in more strong binding of the phage to cellular receptors due to avidity effect, which provokes receptor dimerization and clustering $[3,15,16]$. Another type of tumor selective phagemid gene-delivery vehicles known as phagemid infective particle was designed by [17] using a phagemid system. These particles, $\sim 700 \mathrm{~nm}$ long by $\sim 10 \mathrm{~nm}$ in diameter, display tumor specific pVIII or pIII-fusion peptides which encapsulate a foreign phagemid DNA harboring all genetic elements necessary for expression of transgenes in mammalian cells [9]. Thus, the phage-targeted phagemid gene delivery can be considered as a new to tumor management strategy. In the light of this, we have screened a $1 \times 10^{9}$ clone landscape phage library to isolate metastatic prostate cancer-specific phage clones. The penetration of the most specific phage clone into mammalian cells was studied using fluorescence and electron microscopy. Thereafter, the phage clone was converted into phagelike particles using the phagemid system and its PC-3M cell-transforming activity was observed using emerald green fluorescent protein marker.

\section{Results and discussion}

Selection of prostate cancer cell-interacting phage clones and studying their selectivity towards the target cells

To generate peptides specific for prostate cancer cells, landscape phage library with an octapeptide insert was screened against the metastatic prostate cancer cell line PC-3M involving two selection rounds. 18 clones were randomly picked and the structures of the displayed peptides were determined using PCR, sequencing and translation of the DNA sequences into amino acids using DNASTAR software (DNASTAR, Madison, WI) (Table 1). Phage clone EPTHSWAT was the most
Table 1 Phage clones generated from the second round screening of the $f 8 / 8$ landscape phage library

\begin{tabular}{ll}
\hline Fusion peptides & Frequency \\
\hline EPTHSWAT & 8 \\
GDIVTSNS & 7 \\
DSNGTSTQ & 1 \\
EDEEPSTS & 1 \\
DVTTGAYS & 1 \\
\hline
\end{tabular}

Phage clones are designated by the structure of the inserted (fused) guest peptides.

frequently selected, occurring 8 times, while phage clone GDIVTSNS occurred 7 times.

Selectivity of the two abundant prostate cancerspecific phages EPTHSWAT and GDIVTSNS towards $\mathrm{PC}-3 \mathrm{M}$ cells were studied in a phage capture assay (Figure 1). Selectivity assay is based on interaction of phage particles with $\mathrm{PC}-3 \mathrm{M}$ cells in comparisons with other cells RWPE-1 (non-neoplastic prostate cells), HT29 (human colon carcinoma cells) and serum. As seen in Figure 1, phage EPTHSWAT showed more selective interaction with PC-3M cells with 14.4 fold greater interaction than the phage GDIVTSNS and 35.1 fold greater than the non-relevant control phage VPEGAFSS. Furthermore, phage EPTHSWAT showed a statistically significant higher interaction with PC-3M cells than with other cells RWPE-1, HT-29 and serum. This high interaction of the phage particles with $\mathrm{PC}-3 \mathrm{M}$ cells might be due to phage interaction with overexpressed macromolecules on the surfaces of the cells.

\section{Selective interaction of phage EPTHSWAT in a co-culture with PC-3M, RWPE-1 and HT-29 cells}

To further confirm the selective interaction of phage EPTHSWAT with PC-3M cells;PC-3M, RWPE-1 and HT-29 cells were co-cultured and incubated with phage EPTHSWAT for $1 \mathrm{~h}$ at $37^{\circ} \mathrm{C}$. Phage was fluorescent labeled with antibodies and observed by fluorescence microscopy.

We observed that majority of the phage particles localized in PC-3M cells with very scanty amount in RWPE-1 and HT-29 cells. As shown in Figure 2, the predominant interaction of the phage particles with PC-3M cells is auspicious for harnessing the phage peptides to navigate therapeutics to the desired site of lesion with minimal bystanders' effect.

The selective interaction of phage EPTHSWAT with PC-3M cells in comparison with the non-relevant phage VPEGAFSS at $15 \mathrm{~min}$ or $1 \mathrm{~h}$ was also studied (Figure 3). At 15 min post incubation, phage EPTHSWAT was already interacting with PC-3M cells while the control phage particles were washed away during the washing stage of the procedure. At $1 \mathrm{~h}$ post-incubation, a great 

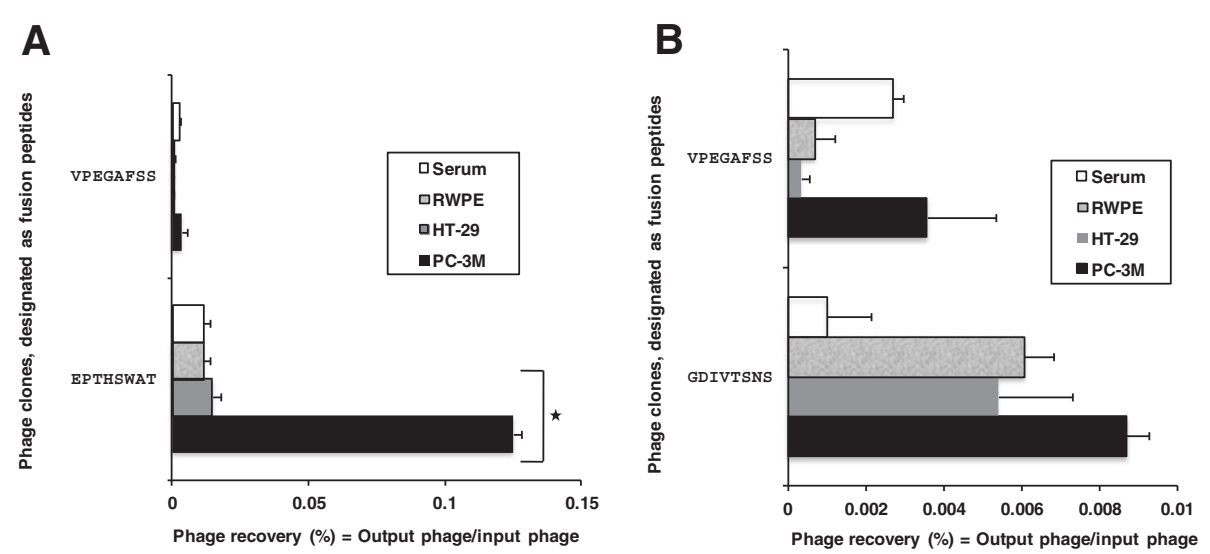

Figure 1 Selectivity of the phage clones EPTHSWAT and GDIVTSNS toward target cells PC-3M in comparison with other cells (nonneoplastic prostate epithelial cells, RWPE-1; colon adenocarcinoma cells, HT-2A) and serum. Panel A depicts levels of interaction of phage EPTHSWAT with PC-3M cells, RWPE cells, HT-29 cells and serum; Panel B shows the levels of phage GDIVTSNS interaction with PC-3M cells, RWPE cells, HT-29 cells and serum. Selectivity was estimated as phage recovery (\%) = output (cell-associated) phage/input phage. Unrelated phage bearing the guest peptide VPEGAFSS was used as a control.

amount of phage EPTHSWAT were already bound or internalized by PC-3M cells whereas very scanty control phage particles were observed interacting with the cells. The interaction of phage EPTHSWAT with the cells at the two time points were specific because phage particles still interacted with the cells despite stringent washing steps employed in the procedure. This is also evidenced by the washing away of majority of the control phage particles bound to the cells through non-specific interactions.

\section{Tracking phage EPTHSWAT interaction with PC-3M cells} Immunogold electron microscopy was employed to precisely track the kinetics of phage EPTHSWAT interactions with PC-3M cells in comparison with the control phage VPEGAFSS.Phage EPTHSWAT was incubated with PC-3M cells at three time points $15 \mathrm{~min}, 1 \mathrm{~h}$ or $24 \mathrm{~h}$. As seen in Figure 4, at 15 min post-post incubation, phage EPTHSWAT particles had already bound to the surface of the cells and some had gain entry into the cytoplasm, whereas, the control phage particles were not observed interacting with the cells.

The binding and internalization of phage EPTHSWAT particles at this time point corroborates the previously described results of immunofluorescence tracking of phage interaction with $\mathrm{PC}-3 \mathrm{M}$ cells. This further revealed the selective interaction of the test phage interacting with $\mathrm{PC}-3 \mathrm{M}$ cells in comparison with the control phage. Absence of the control phage particles interacting with PC-3M was as a result of the washing step in the procedure whereby non-specific interacting phage particles were washed off. The internalization of phage EPTHSWAT into the cytoplasm at 15 min post-incubation might be applicable for harnessing the phage fusion peptides for rapid targeted delivery of therapeutics. Based on the binding and internalizing of the test phage at $15 \mathrm{~min}$ postincubation, it could be inferred that the phage mechanism

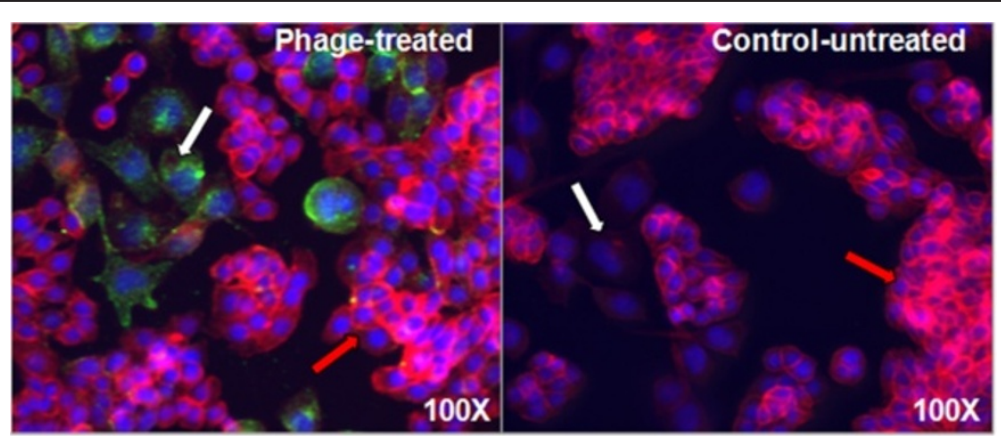

Figure 2 Immunofluorescence microscopic demonstration of phage clone EPTHSWAT selectivity towards PC-3M cells in a co-culture of PC-3M, RWPE-1 and HT-20 cells. White arrows point PC-3M (prostate cancer) cells whereas red arrows point to HT-29 (colon cancer) cells. Phage EPTHSWAT intra-cytoplasmic and nuclear localization at $1 \mathrm{~h}$ post-incubation with the co-culture of PC-3M and HT-20 cells. Phage particles stained with goat anti-rabbit Alexa Fluor 488 were revealed as abundant green spots in PC-3M cells in comparison with the very scanty phage particles in HT-29 cells. The control uninfected co-culture showed no green spots. 


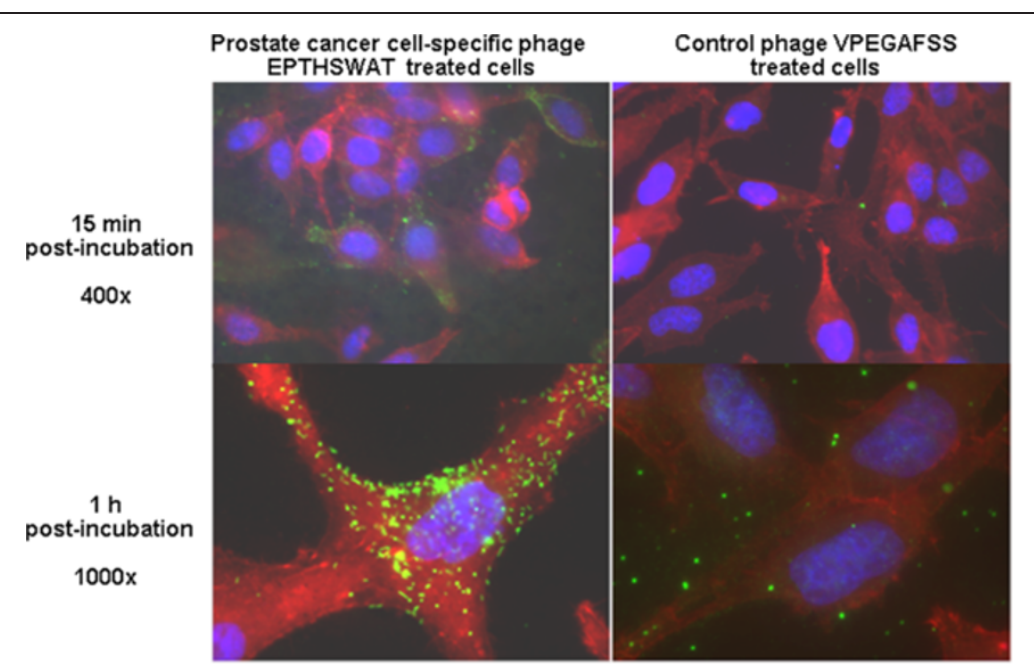

Figure 3 Immunofluorescence microscopic demonstration of phage clone EPTHSWAT interaction with PC-3M cells at two time points $15 \mathrm{~min}$ or $1 \mathrm{~h}$ in comparison with the control non-relevant phage VPEGAFSS. Phage particles were revealed as green spots and linear particles in the cytoplasm and nuclei of the cells at 40X and 100X magnification of the objective lens at 15 min and $1 \mathrm{~h}$ post-incubation, respectively.

of entry into PC-3M cells is not through endocytic pathway. This is also supported by the fact that the length of the phage particle $(1 \mu \mathrm{m})$ will not fit into the membrane pits $700 \mathrm{~nm}$ in depth [18].

\section{Generation of phage-like particles}

To generate phage-like particles, K91 blue E. coli cells were transformed with pcDNA ${ }^{\mathrm{sm}} 6.2$-GW/EmGFP-miR vector encoding emerald green fluorescent protein (EmGFP). The phagemid encodes EmGFP under the control of a Pol II human CMV (cytomegalovirus) promoter and Herpes simplex virus (HSV) thymidine kinase (TK) polyadenylation signal; f1, pUC and SV40 origins of replication; spectinomycin (aadA1) and blasticidin (bsd) resistance genes. The phagemids were rescued with phage EPTHSWAT (helper phage) as illustrated (Figure 5).

\section{Transmission electron microscopic imaging and} quantification of phage-like particles and phage

Transmission electron microscopy (TEM) was employed to visualize the mixture of phage-like particles and phage EPTHSWAT, quantify the particles and estimate their lengths. As anticipated, some phage EPTHSWAT were produced along with the phage-like particles but the number of phage-like particles superseded that of the phage particles due to the vector low copy number and defective assembly of the phage particles created by disruption of the origin of the minus-strand synthesis with the insertion of the Tn10 fragment [19]. The number of phage-like particles on the electron microscopic field was 59 whereas the phage particles were36; therefore the molecular ratio of phage-like particles to the phages is 1.6 as shown (Figure 6).
Virions $/ \mathrm{ml}=\left(\mathrm{A}_{269}\left(6 \times 10^{16}\right) /\right.$ nucleotides in the genome, where $A_{269}$ represents an absorbance at $269 \mathrm{~nm}$ [20]. Therefore, for phage-like particles with the genome size 5759 nucleotides, $1 \quad \mathrm{AU}_{269}=1.04 \times 10^{13}$ virions $/ \mathrm{ml}$ whereas for the phage EPTHSWAT with genome size 9198 nucleotides, $1 \mathrm{AU}_{269}=6.5 \times 10^{12}$ virions $/ \mathrm{ml}$. The molecular ratio of phage-like particles to phage particles is 1.6, as determined by TEM (Figure 6). Thus their mass ratio is $(5759 \times 1.6) / 9198=1$. Thus, $1 \mathrm{AU}_{269}$ of preparation contains $0.5 \times 1.04 \times 10^{13}=5.2 \times 10^{12}$ virions $/ \mathrm{ml}$ of phagelike particles and $0.5 \times 6.5 \times 10^{12}=3.25 \times 10^{12}$ virions $/ \mathrm{ml}$ of phage particles. The phage-like particle is about $2 / 3^{\text {rd }}$ the size of the phage, since the calculated length of the phage-

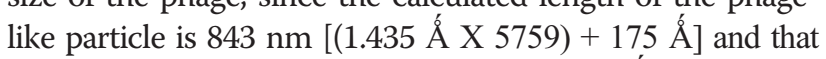
of phage EPTHSWAT is $1337 \mathrm{~nm}[(1.435 \AA$ X 9198) + $175 \AA ̊ x][21]$ as confirmed (Figure 6).

\section{in vitro expression of emerald green fluorescent protein gene}

To track the expression of expression of emerald green fluorescent protein gene in the phage-like particles; phage-like particles in combination with the phage were incubated with $\mathrm{PC}-3 \mathrm{M}$ cells at $37^{\circ} \mathrm{C}$ for $48 \mathrm{~h}$ while cells incubated with phage only served as the control. Cells were washed with PBS, fixed and visualized by epifluorescence microscopy. As seen in Figure 7, some cells incubated with phage-like particles and phage demonstrated emerald green fluorescent protein expression as revealed by the green fluorescence when observed with FITC filter of the epifluorescent microscope (1A) whereas no fluorescence was observed with the control $(2 \mathrm{~A})$. 


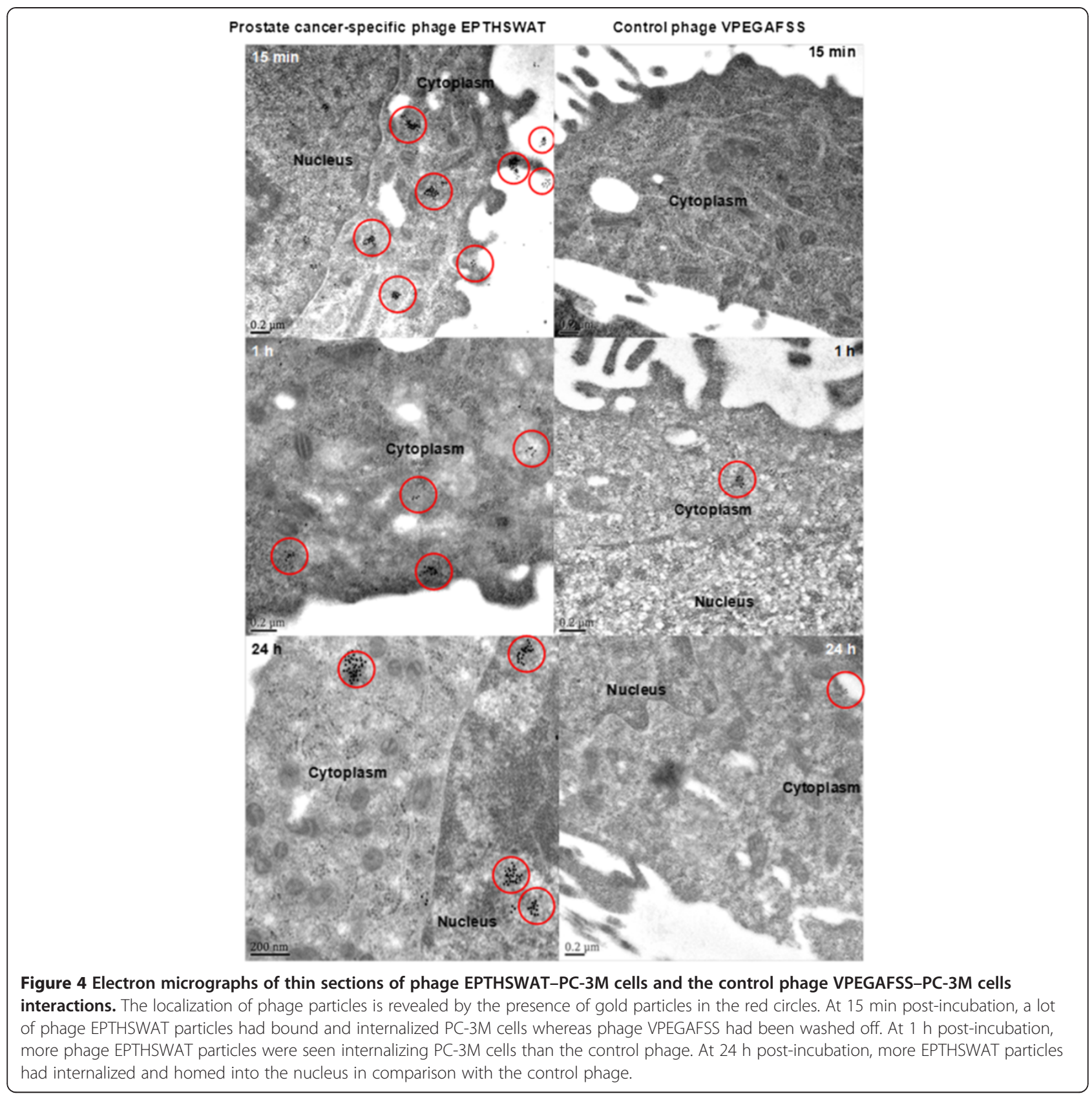

\section{Conclusion}

In this study, we demonstrated that screening of a $2 \times 10^{9}$ landscape phage library against metastatic prostate cancer cell PC-3M allows isolating highly selective prostate cancer cell-specific phage, which can be readily converted into a phage-like particle transducing the mammalian prostate tumor with the potential to deliver therapeutic genes and their expression products to cancer cells leaving normal cells unscathed. Notwithstanding, the procedure was carried in vitro and peptide selectivity to towards prostate tumor has not been tested in vivo; the peptides generated from the screening procedure might home tumor cells when tested in vivo based the stringent steps involved in the selection procedure. Furthermore, peptides generated from screening landscape phage library proffers avidity effect based on multivalency that can arouse receptor dimerization and clustering $[3,15,16]$ in comparison with commonly screened p-III fusion libraries with affinity features. The delivery and expression of emerald green fluorescent protein was revealed by visualization of green fluorescence observed in some of the treated PC-3M cells. Although the expression of the reporter gene EmGFP was not high; this could have been improved if adenoassociated virus inverted terminal repeats were present in 


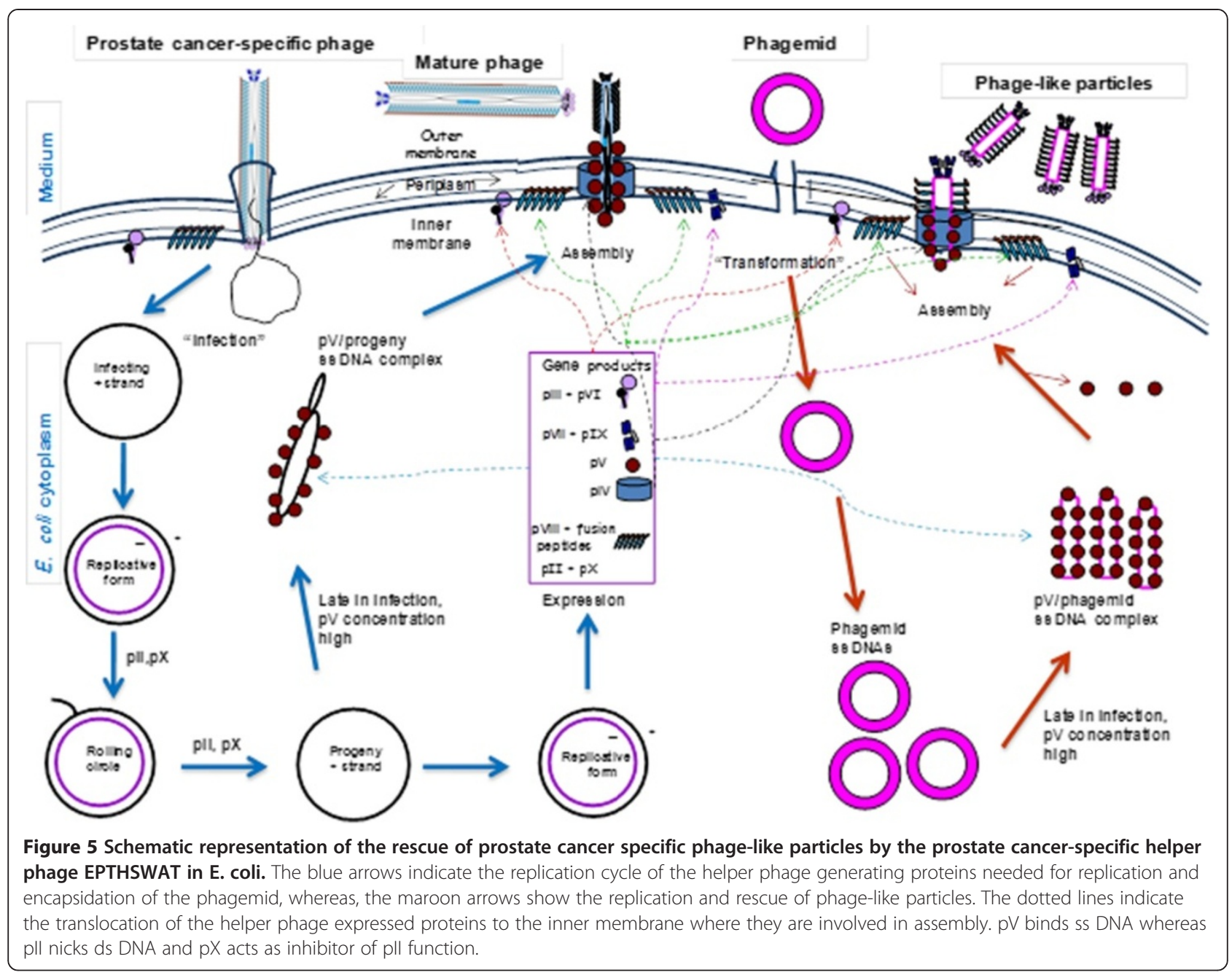

the phagemid vector as demonstrated [8]. The results of this study can pave a way for developing highly efficient transgene or oligonucleotides delivery systems against other maladies aside cancer. Screening of landscape phage libraries provides an effective way for development of highly selective ligands to a variety of cell receptors and their easy conversion into non-pathogenic phage-like particles, which are promising vectors for delivery of various gene pharmaceuticals.

\section{Methods}

\section{Cell lines}

All cell lines were purchased from the American Type Culture Collection (ATCC, Manassas, VA, USA).Cell line PC-3M was employed as the target cell, whereas WI-38 (CCL-75) normal human lung fibroblasts were used for depletion of phage library. Cells were cultivated in $25 \mathrm{~cm}^{2}$ cell culture flasks (Corning Inc., Corning, NY) containing their respective media with L-glutamine (Sigma-Aldrich, St. Louis, MO) supplemented with $10 \%$ fetal calf serum (FCS) at $37^{\circ} \mathrm{C}$ in $5 \% \mathrm{CO}_{2}$ cultivated.

\section{Phage display library}

The landscape phage library $\mathrm{f} 8 / 8$ containing $2 \times 10^{9}$ clones [22] was screened to isolate clones binding target prostate cancer cell lines. All procedures for handling phages, including propagation, purification, titering, isolation of phage clones, and isolation of phage DNA have been described [23] Escherichia coli (E. coli) strain K91Blue $\left\{\right.$ Hfr C thilacZ $\Delta$ M15 lac Y::mkh lacI $\left.{ }^{\mathrm{Q}}\right\}$ used for propagating phages was kindly provided by George Smith [24].

Selection of prostate cancer cell-interacting phage clones Screening of the phage library against PC-3M target cells was run in T-25 cell culture flask. Briefly, an aliquot of the phage library containing 100 billion phage particles in serum-free medium was incubated in an empty cell culture flask at $37^{\circ} \mathrm{C}$ for $1 \mathrm{~h}$ to deplete phage particles binding plastic. Unbound phages recovered from the depletion were incubated with pre-incubated serum in cell culture flask at $37^{\circ} \mathrm{C}$ for $1 \mathrm{~h}$ to deplete phage particles binding serum. Thereafter, unbound phage particles 


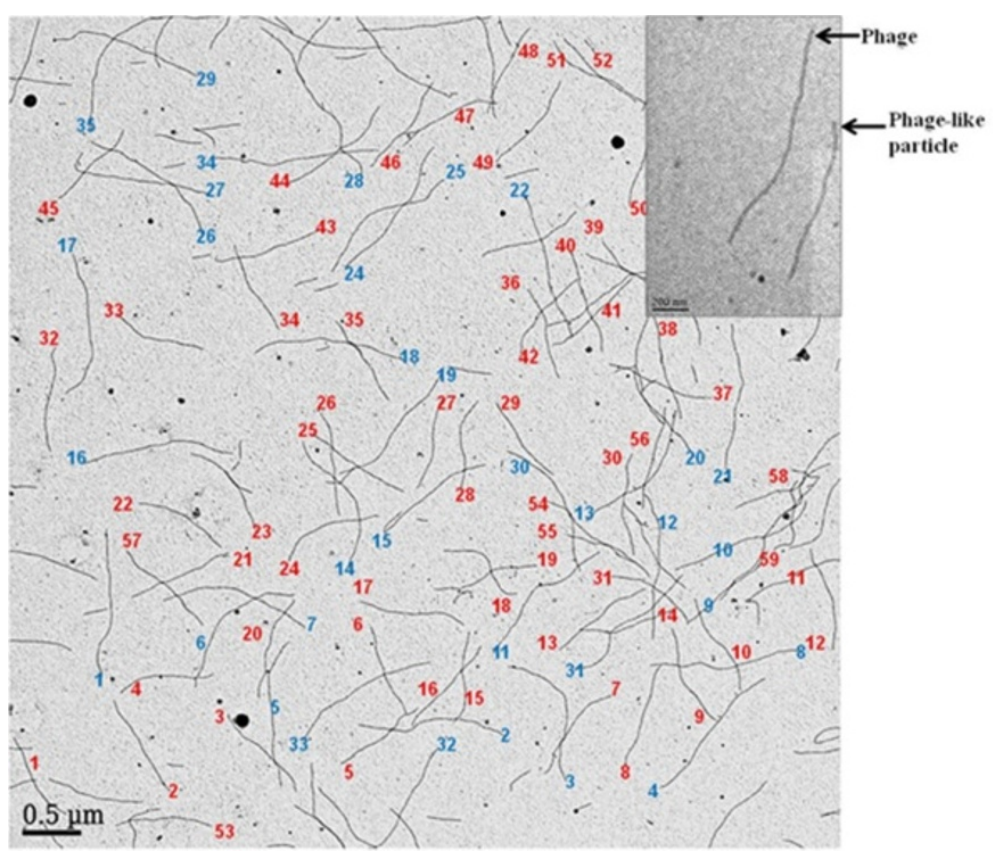

Figure 6 Negative contrast electron micrograph of phage-like particles and phage EPTHSWAT. The phage-like particles are numbered in red whereas the phage particles are in blue. The number of phage-like particles on the shown field is 59 whereas the phage particles are 36 , therefore the ratio of phage-like particles to the phages is 1.6. The phage-like particle is about 2/3rd the size of the phage (Inset).

were incubated with confluent fibroblasts at $37^{\circ} \mathrm{C}$ for $1 \mathrm{~h}$ to deplete phage particles interacting with fibroblasts. Unbound phages were removed, added to the target cells and incubated at $37^{\circ} \mathrm{C}$ for $1 \mathrm{~h}$. Subsequently, unbound phages were discarded and cells were washed ten times with washing buffer $(0.1 \%$ BSA, $0.1 \%$ Tween 20 in serum free medium) to remove any remaining unbound phage particles. Targeted cell-bound phage particles were eluted with elution buffer (0.1 M glycine- $\mathrm{HCl}, \mathrm{pH} 2.2)$ for $10 \mathrm{~min}$ on ice and neutralized with $1 \mathrm{M}$ Tris- $\mathrm{HCl}$ (pH 9.1). Phage particles in eluate were concentrated in Amicon ultra-4 concentrators as recommended by the manufacturer

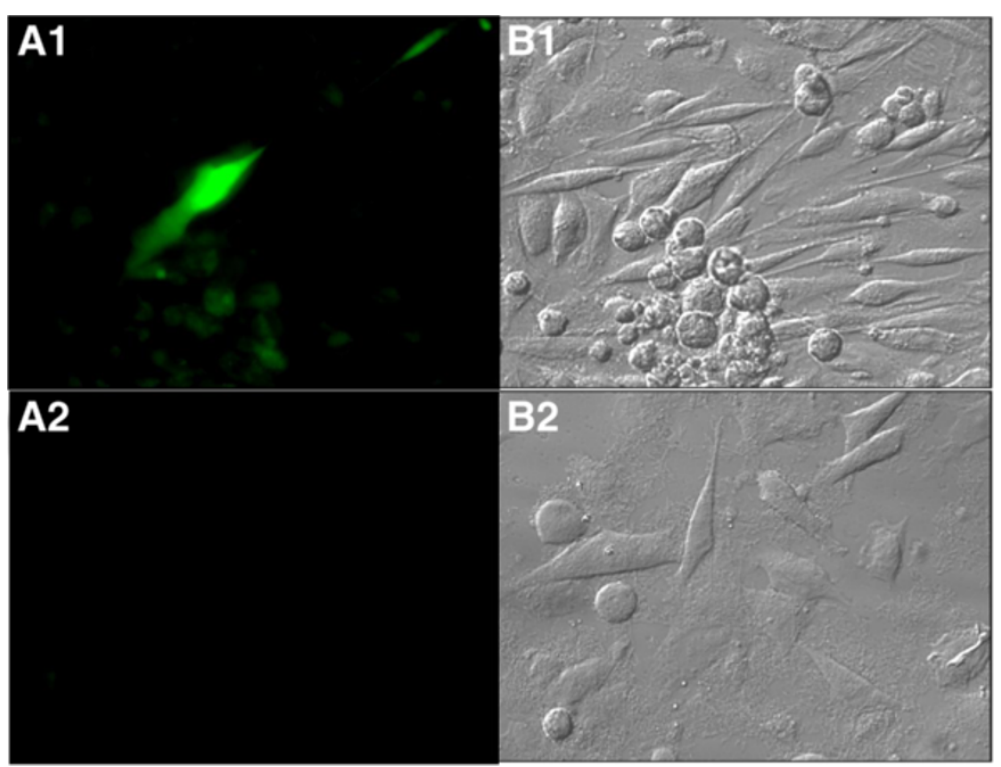

Figure $\mathbf{7}$ in vitro transduction of phage-like particles and expression of EmGFP. A1 and B1 represent PC-3M cells incubated with phage-like particles and phage at $37^{\circ} \mathrm{C}$ for $48 \mathrm{~h}$ and then observed with FITC filter epifluorescence and bright field, respectively. A2 and B2 show cells incubated with phage particles only and observed with FITC filter epifluorescence and bright field, respectively. 
(Millipore Corp, Bedford, MA)and used in subsequent rounds of selection but with no depletion steps. The amplified eluate was as input in further rounds of selection. These procedures were similar to the procedure described above but with the exclusion of the depletion steps depletion steps. Two rounds of selection were performed. In each round, the enrichment of phage particles binding to the cells was determined by titering of input and output phages. The ratio of output to input phage increased from one round to another indicating successful selection of phage clones interacting with the target cell lines.

\section{Testing of phage clones for selectivity towards prostate cancer cells}

PC-3M cell-specific phage clone EPTHSWAT was characterized for their selectivity toward target prostate cancer cell PC-3M in comparison with control cells RWPE (non-neoplastic prostate epithelia), HT-2A (colon carcinoma) and serum in a phage capture assay. Briefly, target cells PC-3M, and control RWPE and HT-2A cells were cultivated in triplicate to confluence in separate wells of 96-well cell culture plates. The medium with serum was incubated in separate wells in triplicate as a control. Cells incubated with serum-free medium at room temperature for $1 \mathrm{~h}$ were incubated with phage $\left(1 \times 10^{6} \mathrm{cfu}\right)$ at room temperature for $1 \mathrm{~h}$. Unbound phages were carefully removed and cells were washed with $100 \mu \mathrm{l}$ washing buffer for $5 \mathrm{~min}$ eight times to remove non-interacting phages. Cells were lysed with $25 \mu \mathrm{l}$ lysis buffer (2.5\% CHAPS) for $10 \mathrm{~min}$ on a shaker. The lysate containing cell-interacting phages were titered in E. coli. Phage recovery was calculated as the ratio of output phage to input phage. An unrelated phage with non-relevant guest peptide VPEGAFSS was used as the control (Figure 1).

\section{Immunofluorescence analysis of the selectivity of phage EPTHSWAT towards PC-3M cells}

PC-3M, HT-29 and RWPE cells were co-cultured in Lab-Tek $^{\text {Tx }}$ 4-well chamber slides (Thermo Scientific, Rochester, NY) until they were $60 \%$ confluent. Subsequently, the cells were incubated with $1 \times 10^{11}$ phage particles in RPMI 1640 medium for $1 \mathrm{~h}$ at $37^{\circ} \mathrm{C}$ while the other cells served as the uninfected controls. Thereafter, cells were washed with PBS (pH 7.4), fixed in 4\% paraformaldehyde for $10 \mathrm{~min}$, permeabilized with $0.25 \%$ Triton X-100 (Sigma, St. Louis, MO) in PBS for $10 \mathrm{~min}$ and blocked with 5\% goat serum (MP Biomedicals, Solon, $\mathrm{OH}$ ) for $1 \mathrm{~h}$. Cells were treated with 1:1000 dilution of $5 \mathrm{mg} / \mathrm{ml}$ rabbit anti-fd bacteriophage antibodies (Sigma-Aldrich, St. Louis, MO) for $1 \mathrm{~h}$, washed and incubated with 1:500 AlexaFlour 488 anti-rabbit IgG, $0.022 \mu \mathrm{M}$ Alexa Flour 546 phalloidin (Molecular Probes,
Carlsbad, CA) for $1 \mathrm{~h}$. Subsequently, cells were washed three times with PBS and covered with VECTASHIELD mounting medium with DAPI (Vector Laboratories, Burlingame, CA). Images were acquired in grayscale with a 12-bit digital camera coupled to Olympus BX51 microscope (Olympus, USA) equipped with bandpass emission fluorescence filter optical blocks. During the processing stage, individual image channels were pseudocolored with RGB values corresponding to each of the fluorophore emission spectral profiles with the exception of Alexa Flour 546 phalloidin, which was pseudocolored red (Figure 2). Interaction of phage EPTHSWAT with PC-3M cells in comparison with the non-relevant phage VPEGAFSS was used to further confirm the selectivity of phage EPTHSWAT towards PC$3 \mathrm{M}$ cells. $1 \times 10^{11}$ phage EPTHSWAT and phage VPEGAFSS were incubated with their corresponding confluent PC-3M cells at $37^{\circ} \mathrm{C}$ for $15 \mathrm{~min}$ or $1 \mathrm{~h}$ and processed as described previously and images were acquired with the Olympus BX51 microscope (Figure 3).

\section{Immunogold electron microscopic analysis of the selectivity of phage EPTHSWAT towards PC-3M cells}

PC-3M cells were seeded into Thermonax cover slips (Thermo Scientific, Rochester, NY) and grown until confluence. $1 \times 10^{11}$ of phage EPTHSWAT particles were incubated with cells at three time points: $15 \mathrm{~min}, 1 \mathrm{~h}$ or $24 \mathrm{~h}$ at $37^{\circ} \mathrm{C}$. For the $24 \mathrm{~h}$ incubation, cell culture growth medium was replaced with new growth medium at $1 \mathrm{~h}$ post-incubation to remove unbound phage particles and cells were thereafter incubated for $24 \mathrm{~h}$. The non-relevant phage VPEGAFSS served as the negative control. At the end of incubation for the three time points, cells were washed thrice with PBS to remove unbound phage particles, fixed in $2 \%$ paraformyladehyde, $1 \%$ glutaraldehyde in $0.1 \mathrm{M}$ cacodylate, $\mathrm{pH}$ 7.4., postfixed in 1\% tannic acid (Electron Microscopic Sciences, Hatfield, PA), dehydrated in ethanol and infiltrated and embedded in LR White resin (Electron Microscopic Sciences, Hatfield, PA). Ultrathin sections were cut on formvar-carbon coated nickelgrids and labeled as described [25] with 1:50 rabbit anti-fd bacteriophage IgG (Sigma-Alrich, St. Louis, MO) and 1:5 goat anti-rabbit conjugated with $10-\mathrm{nm}$ gold particles. Grids were rinsed and post-stained with $0.5 \%$ aqueous uranyl acetate. Samples were observed in Joel 1400 electron microscope operated at $80 \mathrm{kV}$.

\section{Generation of phage-like particles}

$\mathrm{pcDNA}^{\mathrm{m}} 6.2-\mathrm{GW} / \mathrm{EmGFP-miR}$ vector was used to transform electrocompetent K91 blue E. coli and transformants were selected on NZY plates containing $50 \mu \mathrm{g} / \mathrm{ml} \mathrm{spec-}$ tinomycin grown at $37^{\circ} \mathrm{C}$ for $16 \mathrm{~h}$. Transformants were picked and propagated in $2 \mathrm{ml} \mathrm{NZY} \mathrm{media} \mathrm{containing}$ 
$50 \mu \mathrm{g} / \mathrm{ml}$ spectinomycin with shaking at $200 \mathrm{rpm}$ at $37^{\circ} \mathrm{C}$ overnight. Subsequently, $300 \mu \mathrm{l}$ overnight cultures was added to $20 \mathrm{ml}$ of NZY plates containing $50 \mu \mathrm{g} / \mathrm{ml} \mathrm{spec-}$ tinomycin and grown at $37^{\circ} \mathrm{C}$ in a shaking incubator at $200 \mathrm{rpm}$ until the OD reached 0.450 at $600 \mathrm{~nm}$. Thereafter, the culture was inoculated with $1.0 \times 10^{9}$ colony forming units $(\mathrm{cfu})$ of the prostate cancer-selective phage EPTHSWAT and incubated at $37^{\circ} \mathrm{C}$ for $15 \mathrm{~min}$ at $50 \mathrm{rpm}$ for the phage to infect the E. coli. Then, $0.2 \mu \mathrm{g} / \mathrm{ml}$ of tetracycline was added to the culture and incubated at $37^{\circ} \mathrm{C}$ for $40 \mathrm{~min}$ at $200 \mathrm{rpm}$. Following, tetracycline was added to $20 \mu \mathrm{g} / \mathrm{ml}$ and the culture was grown overnight. Finally, the phage-like particles preparation were collected by two successive polyethylene glycol 8000 precipitations and suspended in $100 \mu \mathrm{l}$ Tris buffered saline (TBS; $50 \mathrm{mM}$ Tris- $\mathrm{HCl}$, pH 7.5 and $150 \mathrm{mM} \mathrm{NaCl}$ ).

\section{Transmission electron microscopic imaging and quantification of phage and phage-like particles}

$6 \mu \mathrm{l}$ of a mixture of phage-like particles and phage were dispensed on a carbon film coated copper grid (Electron Microscopic Sciences, Hatfield, PA) and incubated for $5 \mathrm{~min}$ at room temperature. The mixture was tipped off and the grid was stained with a drop of $5 \%$ uranyl acetate for $5 \mathrm{~min}$ at room temperature. The stain was tipped off and the grid was allowed to dry for $5 \mathrm{~min}$ and observed in Joel 1400 electron microscope operated at $80 \mathrm{kV}$. The quantities and ratio of phage-like particles and phage in the preparation were determined by counting their numbers in a field of view as depicted (Figures 6 and 7).

\section{in vitro expression of emerald green fluorescent protein}

To test the expression of emerald green fluorescent protein in PC-3M cells, $8.0 \times 10^{10}$ phage-like particles with $5.0 \times 10^{10}$ phage EPTHSWAT were incubated with confluent PC-3M cells in the well of a Lab-Tek ${ }^{\mathrm{Tm}}$ 4-well chamber slide (Thermo Scientific, Rochester, NY) for 48 $\mathrm{h}$ at $37^{\circ} \mathrm{C}$ while cells incubated with only $5.0 \times 10^{10}$ phage EPTHSWAT served as the control. Cells were washed three times with PBS, fixed, visualized and imaged under Olympus BX51 microscope (Olympus, USA) with FITC emission fluorescence filter and bright field (Figure 7).

\section{Competing interests}

The authors declare that they have no competing interests.

\section{Authors' contributions}

AE was the principal investigator and takes primary responsibility for the paper. VAP and OAF were instrumental in the design of the study. OAF performed the laboratory work for this study. AE, VAP and RAK participated in reviewing and editing the manuscript. All authors read and approved the final manuscript.

\section{Acknowledgement}

Support for this research was provided by funds from the Raymond W. Freese Post-doctoral Fellowship, Cancer Research Center, Columbia, MO

\section{Author details}

${ }^{1}$ Cancer Research Center, Columbia, MO 65201, USA. ²University of Missouri, Columbia, MO 65211, USA. ${ }^{3}$ Department of Pathobiology, College of Veterinary Medicine, Auburn University, Montgomery, AL 36849, USA. ${ }^{4}$ Department of Veterinary Microbiology and Parasitology, University of Ibadan, Ibadan, Nigeria.

Received: 4 June 2013 Accepted: 26 August 2013

Published: 23 September 2013

\section{References}

1. Siegel R, Naishadham D, Jemal A: Cancer statistics, 2013. CA Cancer J Clin 2013, 63:11-30.

2. Carrasco RA, Stamm NB, Marcusson E, Sandusky G, Iversen P, Patel BKR: Antisense inhibition of survivin expression as a cancer therapeutic. Mol Cancer Ther 2011, 10:221.

3. Urbanelli L, Ronchini C, Fontana L, Menard S, Orlandi R, Monaci P: Targeted gene transduction of mammalian cells expressing the HER2/neu receptor by filamentous phage. J Mol Biol 2001, 313:965-976.

4. Douglas KL: Toward development of artificial viruses for gene therapy: A comparative evaluation of viral and non-viral transfection. Biotechnol Prog 2008, 24:871-883.

5. Schaffer DV, Koerber JT, Lim KI: Molecular engineering of viral gene delivery vehicles. Annu Rev Biomed Eng 2008, 10:169-194.

6. Kassner PD, Burg MA, Baird A, Larocca D: Genetic selection of phage engineered for receptor-mediated gene transfer to mammalian cells. Biochem Biophys Res Commun 1999, 264:921-928.

7. Muller OJ, Kaul F, Weitzman MD, Pasqualini R, Arap W, Kleinschmidt JA, Trepel M: Random peptide libraries displayed on adeno-associated virus to select for targeted gene therapy vectors. Nat Biotechnol 2003, 21:1040-1046.

8. Hajitou A, Rangel R, Trepel M, Soghomonyan S, Gelovani JG, Alauddin MM, Pasqualini R, Arap W: Design and construction of targeted AAVP vectors for mammalian cell transduction. Nat Protoc 2007, 2:523-531.

9. Petrenko V: Evolution of phage display: from bioactive peptides to bioselective nanomaterials. Expert Opin Drug Deliv 2008, 5:825-836.

10. Larocca D, Kassner PD, Witte A, Ladner RC, Pierce GF, Baird A: Gene transfer to mammalian cells using genetically targeted filamentous bacteriophage. Faseb J 1999, 13:727-734.

11. Poul MA, Marks JD: Targeted gene delivery to mammalian cells by filamentous bacteriophage. J Mol Biol 1999, 288:203-211.

12. Larocca D, Baird A: Receptor-mediated gene transfer by phage-display vectors: applications in functional genomics and gene therapy. Drug Discov Today 2001, 6:793-801.

13. Hajitou A, Trepel M, Lilley CE, Soghomonyan S, Alauddin MM, Marini FC, Restel BH, Ozawa MG, Moya CA, Rangel R, Sun Y, Zaoui K, Schmidt M, von Kalle C, Weitzman MD, Gelovani JG, Pasqualini R, Arap W: A hybrid vector for ligand-directed tumor targeting and molecular imaging. Cell 2006, 125:385-398.

14. Li Z, Zhang J, Zhao R, Xu Y, Gu J: Preparation of peptide-targeted phagemid particles using a protein III-modified helper phage. Biotechniques 2005, 39:493-497.

15. Ivanenkov W, Felici F, Menon AG: Targeted delivery of multivalent phage display vectors into mammalian cells. Biochim Biophys Acta 1999, 1448:463-472.

16. Legendre D, Fastrez J: Construction and exploitation in model experiments of functional selection of a landscape library expressed from a phagemid. Gene 2002, 290:203-215.

17. Mount JD, Samoylova TI, Morrison NE, Cox NR, Baker HJ, Petrenko VA: Cell targeted phagemid rescued by preselected landscape phage. Gene 2004, 341:59-65.

18. Willingham MC, Pastan I: The receptosome: An intermediate organelle of receptor-mediated endocytosis in cultured fibroblasts. Cell 1980, 21:67-77.

19. Smith GP: Filamentous phage assembly: morphogenetically defective mutants that do not kill the host. Virology 1988, 167:156-165. 
20. Barbas CF, Scott JK, Silverman GJ: Phage display: a laboratory manual. New York: Cold Spring Harbor Laboratory Press; 2001.

21. Makowski L: Structural constraints on the display of foreign peptides on filamentous bacteriophages. Genes 1993, 128:5-11.

22. Petrenko VA, Smith GP, Gong X, Quinn T: A library of organic landscapes on filamentous phage. Protein Eng 1996, 196:797-801.

23. Brigati JR, Samoylova TI, Jayanna PK, Petrenko VA: Phage display for generating peptide reagents. Curr Protoc Protein Sci 2008. Feb; Chapter 18: Unit 18.9. doi: 10.1002/0471140864.ps1809s51.

24. Yu J, Smith GP: Affinity maturation of phage-displayed peptide ligands. Methods Enzymol 1996, 267:3-27.

25. Thorpe JR: The application of LR gold resin for immunogold labeling. Methods Mol Biol 1999, 117:99-110.

doi:10.1186/1477-3155-11-31

Cite this article as: Fagbohun et al:: Metastatic prostate cancer cellspecific phage-like particles as a targeted gene-delivery system. Journal of Nanobiotechnology 2013 11:31.

\section{Submit your next manuscript to BioMed Central and take full advantage of:}

- Convenient online submission

- Thorough peer review

- No space constraints or color figure charges

- Immediate publication on acceptance

- Inclusion in PubMed, CAS, Scopus and Google Scholar

- Research which is freely available for redistribution 